\title{
Iberian seismicity, 1961 - 1965
}

\author{
J. M. Muntera
}

Ricevuto il 5 Novembre 1966

\begin{abstract}
SUMmany. - This paper compares the seismic results corresponding to 1961-65 with those presented in several author's prior studies for the Iberian Peninsula region, which were obtained reducing to a 50 year period the available seismic material up to 1960 . The present paper lists 237 earthquakes, a selection between those occurred and recorded; their epicentral coordinates, origin time, depth and magnitude from body waves; and a discussion about the seismically active quadricles on the area, regional density, epicenter display, annual frequency in steps of $1 / 4 \mathrm{~m}$, the parabolic adjustment for $\log \lambda, m$, tectonic flux and intermediate foci location related with the main tectonic features.

1961-65 is a tenth of the foresaid reduced 50 Y.P. and the present results agree with the preliminary ones, as it was expected; mainly for the following features: a) migration of maximal density which is Easterly; b) a satisfactory parabolic regression for $\log N, m ; c)$ the cyclic appearance regarding annual frequency variation; $d$ ) continuation of the accumulative behaviour for the strain release curve during the present period, which began in $1954 ; e$ ) seismicity is significatively of an intralpine character, and $f$ ) intermediate foci (preliminary determinations) laying out by line with both: Guadalquivir Fault, SW extension, and Alboran Sea's bottoms.
\end{abstract}

Resuren. - Este artículo compara los resultados correspondientes a 1961-65 con los presentados en otros anteriores trabajos del autor para la Región de la Peníusula Ibérica, los cuales se dedujeron reduciendo a un período de 50 años el material disponible hasta 1960. Se da una lista con 237 terremotos, una selección entre los ocurridos y registrados; sus coordenadas epicentrales, tiempo origen profundidad y magnitud deducida de ondas internas, y se analizan las cuadrículas activas del área, la densidad regional, la situación de los epicentros, la frecuencia anua en grupos de $1 / 4 m$, el ajuste parabólico entre $\log N$ y $m$, el flujo tectónico y la localización de focos intermedios relacionada con los rasgos más salientes de la tectónica.

1961-65 es un décimo del antedicho período de 50 años y los presentes resultados concuerdan con los preliminares, como se esperaba, mayormente 
en lus siguientes caracteres: a) migración de la densidad máxima desde el $\mathrm{L} ; b)$ satisfactoria regresión parabólica de $\log N, m ; c)$ apariencia cíclica en la variación de la frecuencia ánua; $d$ ) continuación del comportamiento acumulativo durante el presente período y para la curva de liberación do esfuerzos, que comenzó en 1954; e) la sismicidad tiene acusado caracter intralpino, y f) los focos intermedios (determinaciones preliminares) caen a lo largo de la Falla del Guadalquivir, extendida al SW, y de los fondos más profundos del mar de Alborán.

RIASSUNTo. - I'autore confronta i risultati relativi alla sismicità della Penisola Iberica dal 1961 al 1965, con quelli precedentemente pubblicati cla altri Autori e ricavati da dati sismici ottenuti in 50 anni, fino al 1960 .

In questa nota vengono riportati in una lista di 237 terremoti (fra quelli avvenuti e quelli registrati), le coordinate epicentrali; il tempo origine; le profonditì e magnituto dedotte dalle onde spaziali; una discussione sulla quadricle sismicamente attive nella zona presa in esame; la posizione degli epicentri; la frequenza annuale in gruppi di $1 / t m$, l'accordo parabolico fra $\log N$ ed $m$; il flusso tettonico, e infine la posizione dei fuochi intermedi riferiti alle principali linee tettoniche.

I risultati ottenuti nel periodo $1961-1965$ concordano - come del resto si sperava - - con quelli del cinquantennio già in precedenza studiato, specialmente per le seguenti caratteristiche: a) migrazione della densità massima verso Est; $b$ ) una soddisfacente regressione del $\log N$ ed $m$; c) una apparenzi ciclica della variazione anmuale della frequenza; $d$ ) la continuiti dell'andamento cumulativo della curva degli sforzi liberati nel periodo preso in esame, ed iniziato nel 1954; e) il carattere intralpino della sismicitì; f) la posizione dei fuochi intermedi (determinazione preliminare), i quali cadono fra la faglia del Guadalquivir (al largo) verso SW, e i fondali molto profondi del Mare di Alboran.

\section{Introduction.}

The purpose of this paper is to check the present seismic results, 1961-1965, with those deduced for the " 50 year period" (") regarding the Iberian Peninsula region, which extends $1.3 \pm 0,000 \mathrm{sq} \mathrm{km}$, approximately.

The material used is (quoted in the "Boletín de sismos próximos" (**) wherein issues the preliminary determinations of epicentral coordinates, origin time, focal depth and magnitude of the earthquakes occurred inside the area $35^{\circ}-44^{\circ} \mathrm{N}, 10^{\circ} \mathrm{W}-5^{\circ} \mathrm{E}$. Then, you have five years

(*) See references CM, SD, EF, PM, ES, IIA.

$\left({ }^{* *}\right)$ Which is published by the Laboratorio Central des Servicio de Sismología. 
of instrumental data, one tenth to compare again the foresaid 50 Y.l., which was established reducing all material available up to 1960.

\section{JOCATED SHOCKS.}

Table I lists the earthquakes with epicenters located on the area during the lapse 1961-1965, using the records from the Spanish peninsular seismological stations and also the information from the neighbouring Portuguese, Algerian and Morocco ones. Table II quotes the remarks corresponding to Table I. They were located 237 shocks, $16.6 \mathrm{per}$ eent between 1453 earthquakes in the Boletin; this pereentage represents a progress regarding acceptable location, as follows:

\begin{tabular}{|c|c|c|c|c|}
\hline Lapse, years & $\begin{array}{c}\text { Unlocated } \\
\text { shocks }\end{array}$ & $\begin{array}{c}\text { Llocaled shocks } \\
\text { conjectural }\end{array}$ & Acceptable & Remarks \\
\cline { 1 - 2 } $1801-1900$ & 38.9 & 60.6 & 0.5 & (2) historical \\
$1901-1950$ & 68.8 & 23.6 & 7.6 & $(2)$ instrumental \\
$1951-1960$ & 85.5 & 6.9 & 7.6 & $(2)$ idem \\
$1961-1965$ & 83.4 & 0.9 & 15.7 & idem \\
\hline
\end{tabular}

Table I and Table II are prepared as it was made in $S D$ and $E F$, completing to date the summary for the earthquakes located on the area through 2365 years. Column $\mathrm{k}$ (kind) indicates the quality of data,

$R$ recording data, but no complete seismic information;

$E$ macro- and micro- epicentral determination;

II micro-determination and also magnitude from records;

$C$ id. id. id. and epicentral intensity;

$B$ id. id. id. and numerical error in $H$, processing a computer program (*).

Only nine earthquakes are of $B$ kind: No. 3310, 3326, 3341, 3436, $3447,3454,3455,3507$ and 3527 . These results have been deduced

(*) The program computes the standard deviation in $I I$ for each $h$ and epicentral coordinates as input, up to a limit of $1000 \mathrm{~km}$ for epicentral distance. 
'lable I - LIS' OF TIE EARTIQUAKES LOCATED ON TIIE AREA.

Seventh epoch. 5 years.

\begin{tabular}{|c|c|c|c|c|c|c|c|c|c|c|c|c|}
\hline Order & li. & year & date & $I I\left(\mathrm{U}^{\prime} \mathrm{T}\right)$ & Lat $\mathrm{N}$ & Long & h & $m$ & Int. Iocalities & \multicolumn{2}{|c|}{ Notes } & Map \\
\hline 3308 & C & 1961 & .J & 101250 & 37.0 & -3.7 & $n$ & 4.1 & II Granada & & & D13 \\
\hline 3309 & II & 1961 &.$J \quad 13$ & 031303 & 37.3 & -3.5 & 45 & 4.1 & Atarfe & & & E 13 \\
\hline 3310 & B & 1961 & J 19 & 163327 & 37.0 & -6.2 & 113 & 4.9 & IV s. Lucar & & (.) & D08 \\
\hline 3311 & $\mathrm{C}$ & 1961 & 110 & 185203 & 41.6 & -5.8 & 33 & 5.2 & V Zamora & $6 . \mathrm{ml}$ & (.) & S09 \\
\hline 3312 & $M$ & 1961 & MIII & 082911 & 36.3 & --7.1 & n & 4. & G. Cadliz & & & $\mathrm{Cos}$ \\
\hline 3313 & $\mathrm{C}$ & 1961 & II 16 & 073039 & 38.1 & -1.1 & $\mathrm{n}$ & 4.1 & III Fortuna & & & $\mathrm{J} 18$ \\
\hline 3314 & $\mathbf{R}$ & 1961 & A 5 & 065906 & 42.5 & -4.8 & & & Santander & (BCIS) & & WV11 \\
\hline 3315 & $M$ & 1961 & $\Lambda 9$ & 133328 & 37.5 & -0.3 & 11 & 4.3 & ( Palos & & & $\mathrm{E} 20$ \\
\hline 3316 & $\mathrm{C}$ & 1961 & A 14 & 162452 & 38.0 & -1.2 & n & 4.3 & III Murcia & 1. & & 1118 \\
\hline 3317 & II & 1961 & Y 29 & 052727 & 36.1 & -0.7 & n & 4.7 & Algeria & & & C19 \\
\hline $33 \mid 8$ & $\mathrm{R}$ & 1961 & J. 4 & 024048 & 38.0 & -9.0 & & & Portugal & (BCIS) & & 1102 \\
\hline 3319 & $\mathrm{C}$ & 1961 & I 12 & 141446 & 37.3 & -3.7 & n & 4.7 & IV P. Puente & 2. & & E13 \\
\hline 3320 & $M$ & 1961 & $\mathrm{~L} 22$ & 050922 & 36.7 & -5.4 & $n$ & 4.5 & Ebrique & & & D 10 \\
\hline 3321 & c & 1961 & L 31 & 222833 & 37.3 & -3.7 & n & 4.4 & V Albolote & & (.) & E 13 \\
\hline 3322 & $M$ & 1961 & (i) 8 & 10 & 44.0 & -7.3 & 11 & 4.9 & Vivero & & & $\mathrm{X} 06$ \\
\hline 3323 & MI & 1961 & ( $\times 25$ & 195950 & 36.3 & -2.6 & n & 4. & Almeria & & & $\mathrm{C} 15$ \\
\hline 3324 & C & 1961 & S 3 & 233314 & 41.9 & -2.5 & n & 4.9 & VII Agreda & 18. $\mathrm{III}$ & $()$. & S15 \\
\hline 3325 & MI & 1961 & S 11 & 071916 & 36.8 & -9.2 & n & 4.8 & S. Vicente & & & D02 \\
\hline 3326 & $\mathrm{~B}$ & 1961 & S 28 & 222552 & 36.3 & --8.0 & 113 & 5.0 & "S. Maria & & (.) & $\mathrm{Co4}$ \\
\hline 3327 & c & 1961 & 06 & 032032 & 36.5 & +1.2 & 11 & 4.9 & VI Orleansville & & $()$. & $\mathrm{C} 23$ \\
\hline 3328 & II & 1961 & 024 & 195 & 37.7 & -4.0 & 11 & 4.1 & Martos 0 & & & III 2 \\
\hline 3329 & $\mathrm{C}$ & 1961 & $N 12$ & 113957 & 37.5 & -3.5 & n & 4.5 & IV Albolote & & & $\mathrm{E} 13$ \\
\hline 3330 & $\mathrm{E}$ & 1961 & N 23 & 044536 & 36.5 & +2.8 & 11 & & V Joinville & (BCIS) & & $\mathrm{C} 26$ \\
\hline 3331 & MI & 1961 & D 8 & 1053 & 38.3 & -1.9 & $n$ & 4.8 & & & & 1119 \\
\hline 3332 & $M$ & 1961 & D 22 & 1200 & 41.8 & -5.6 & 11 & 4. & $\mathrm{Za}$ & 2. & & So9 \\
\hline 3333 & $\mathbf{R}$ & 1962 & & 14 & 36.4 & -3.3 & n & & Alboran & & & $\mathrm{Cl} 4$ \\
\hline 3334 & $\mathrm{R}$ & 1962 & J 13 & 093037 & 36.1 & -3.3 & 11 & & Albo & & & $\mathrm{C} 14$ \\
\hline 3335 & M & 1962 & J 13 & 09 & 5.6 & -3.3 & n & 4. & Al & & & $\mathrm{B} 14$ \\
\hline 3336 & M & 1962 & J 14 & 0445 & 36.4 & -3.4 & n & 4. & Mo & & & $\mathrm{Cl} 4$ \\
\hline 3337 & M & 1962 & J 15 & 044804 & 36.9 & -3.5 & n & 3.6 & $\mathrm{Or}_{\mathrm{r}}$ & & & D13 \\
\hline 3338 & MI & 1962 & J 15 & 1649 & 36.4 & -3.4 & 11 & 4.5 & Motril & 1. & & $\mathrm{C} 13$ \\
\hline 3339 & $\mathrm{M}$ & 1962 & J 17 & 235323 & 37.3 & -3.1 & 11 & 4.1 & Guadix & & & E 14 \\
\hline 3340 & $\mathrm{M}$ & 1962 & J 23 & 033050 & 37.5 & -4.5 & 11 & 4.4 & Cabra & & & Ell \\
\hline 334 & B & 1962 & J 28 & 03242 & 36.3 & -7.8 & 95 & 4. & G. ( & & & $\mathrm{COG}$ \\
\hline 3342 & $\mathrm{C}$ & 1962 & F 11 & 133246 & 37.3 & -2.1 & $n$ & 4.6 & IV Vera & & & E 16 \\
\hline 3343 & (: & 1962 & F 14 & 1348 & 35.4 & -3.1 & $n$ & 4.8 & III Melill & 2. & & Al4 \\
\hline 3344 & $M$ & 1962 & F 21 & 090241 & 35.5 & -3.4 & n & 4. & Melilla & & & Al4 \\
\hline 3345 & M & 1962 & F 21 & & & -3.1 & n & 3.8 & Adra & & & $\mathrm{Cl} 4$ \\
\hline $33+6$ & MI & 1962 & M I & 221955 & 35.8 & -3.6 & 25 & 4.9 & Alboran & 1. & (.) & B13 \\
\hline 3347 & $\mathrm{R}$ & 1962 & 111 & 234050 & 39.4 & -1.8 & & & II Salobre & & & $\mathrm{L} 17$ \\
\hline 3348 & $M$ & 1962 & ML 20 & 000142 & 38.5 & -5.6 & n & 4.6 & Badajoz & & & L09 \\
\hline 3349 & MI & 1962 & A 2 & 144236 & 36.2 & -2.4 & n & 4. & Alboran & & & $\mathrm{Cl} 6$ \\
\hline 3350 & $\mathrm{M}$ & 1962 & 19 & 085256 & 35.7 & -9.0 & 33 & 5.1 & IV Casablanca & & $()$. & $\mathrm{BO2}$ \\
\hline 33 & $\mathrm{C}$ & 15 & A 1 & & & -2.3 & 11 & & & & & Dl6 \\
\hline 3352 & M & 1962 & A 18 & 084341 & 37.2 & -3.5 & 11 & 3.5 & S. Nevada & & & E 13 \\
\hline
\end{tabular}


(Tab. I, cont.)

\begin{tabular}{|c|c|c|c|c|c|c|c|c|c|c|c|c|}
\hline Order & $k$ & year & date & $I I(\mathrm{UT})$ & Lat $N$ & Long & lı & $m$ & Int. Iocalities & \multicolumn{2}{|c|}{ Notes } & Map \\
\hline 3353 & C & 1962 & A 25 & 213352 & 37.2 & -3.5 & 11 & 4.5 & IV s. Nevada & 1. & $()$. & E 13 \\
\hline 3354 & $\mathrm{C}$ & 1962 & A 26 & 081313 & 37.3 & -3.5 & 11 & 3.8 & 111 Iznalloz & & & E 13 \\
\hline 3355 & $\mathrm{R}$ & 1962 & & 065200 & 42.1 & +2.6 & & & Amer & & & Т26 \\
\hline 3356 & C & 1962 & Y 3 & 232725 & 43.6 & -7.3 & 33 & 5.2 & VI Vivero & $m$ & (.) & $\mathrm{x} 06$ \\
\hline 3357 & MI & 1962 & & 040209 & 36.5 & -3.6 & II & 3.6 & Alboran & & & $\mathrm{Cl3}$ \\
\hline 3358 & $M$ & 1962 & Y 8 & $08354 \mathrm{I}$ & 37.1 & -3.5 & 11 & 3.6 & S. Nevada & & & E13 \\
\hline 3359 & MI & 1962 & $\vec{Y} 12$ & 005202 & 36.7 & -3.5 & n & 3.7 & Motril & & & $\mathrm{D} 13$ \\
\hline 3360 & $\mathrm{C}$ & 1962 & U 1 & 195917 & 36.4 & -3.0 & 11 & 4.3 & II Adra & 3. & $()$. & $\mathrm{Cl}$ \\
\hline 3361 & $\mathrm{C}$ & 1962 & U 9 & 055946 & 42.0 & +0.7 & 11 & 4.7 & $V$ Ribagorzana & 111 & & $\mathbf{S} 22$ \\
\hline 3362 & MI & 1962 & L 15 & 18 & 36.2 & -0.8 & 11 & & & & & C19 \\
\hline 3363 & $\mathrm{MI}$ & 1962 & L 16 & 162156 & 37.3 & -4.7 & 11 & 3.9 & . & & & E 11 \\
\hline 3364 & $\mathrm{R}$ & 1962 & G 14 & 221405 & 36.6 & -6.0 & & & $111 A r \cos$ & & (.) & D08 \\
\hline 3365 & $\mathrm{MI}$ & 1962 & G 14 & 223837 & 36.6 & -6.0 & 11 & 4.3 & Are & 3. & & Dos \\
\hline 3366 & MI & 1962 & G 25 & 195851 & 36.6 & +0.9 & 33 & 4.6 & Algeria & & & D22 \\
\hline 3367 & $\mathrm{R}$ & 1962 & G 26 & 122 & 37.4 & -2.3 & & & Jaen & & (.) & E 14 \\
\hline 3368 & MI & 1962 & G 26 & 163052 & 36.7 & +1.3 & 15 & 4.8 & Algeria & & (.) & $\mathrm{D} 23$ \\
\hline 3369 & MI & 1962 & G 29 & 164853 & 37.2 & -4.1 & 11 & 4 & Ioja & & & EI 2 \\
\hline 3370 & $M$ & 1962 & S 4 & 151144 & 36.8 & -9.0 & 33 & 5.0 & S. Vicente & & (.) & $\mathrm{D} 02$ \\
\hline 3371 & MI & 1962 & S 9 & 014713 & 36.3 & -6.7 & 11 & 3.7 & Vadiz & & & $\mathrm{Co7}$ \\
\hline 3372 & $\mathrm{C}$ & 1962 & S 11 & & & -2.6 & II & & $11 \mathrm{Aln}$ & & & D 15 \\
\hline 3373 & iI & 1962 & S 16 & 1 & .3 & -2.2 & 11 & 4.2 & La Roda & 1. & & L1 6 \\
\hline 3374 & MI & 1962 & S 22 & & .1 & -3.5 & 11 & 3.4 & S. Nevalla & 2. & & 13 \\
\hline 3375 & MI & 1962 & S 27 & 034 & 6 & -3.2 & II & 3.7 & Albuñol & & & 014 \\
\hline 3376 & MI & 1962 & S 28 & & 3 & -3.0 & n & 4 & Ad & & & 114 \\
\hline 3377 & C & 1962 & 08 & 1 & 7 & -1.5 & n & 4.9 & III S. Almeria & & & 017 \\
\hline 3378 & MI & 1962 & O 14 & & & -3.6 & 11 & 3 & Pad & & & 13 \\
\hline 3379 & MI & 1962 & $\begin{array}{ll}0 & 29\end{array}$ & $15222 \mathrm{~S}$ & 37.0 & -3.6 & 11 & 3.5 & & & & 013 \\
\hline 3380 & $\mathrm{R}$ & 1962 & 030 & 070110 & 37.1 & -2.2 & & & Imeria & & $()$. & E 16 \\
\hline 3381 & $\mathrm{C}$ & 1962 & 2 & 180653 & 2.3 & +2.1 & & 5 & V Ri & $2 . m$ & & 25 \\
\hline 3382 & $\mathrm{MI}$ & 1962 & & & 37.5 & -2.8 & II & 4.7 & $\mathrm{~B}$ & & & 15 \\
\hline 3383 & $\mathrm{C}$ & 1962 & N 22 & & 3.2 & -0.5 & 11 & 3.9 & IV Tabarea & $\mathrm{m}$ & & $\mathrm{J} 19$ \\
\hline 3381 & $\mathrm{R}$ & 1962 & D 4 & 13 & .8 & -2.1 & & & C. Gata & & (.) & D)16 \\
\hline 3385 & $\mathrm{C}$ & 1962 & D 31 & & 41.9 & +1.0 & n & 5.0 & IV 'Tremp & 1. & & 522 \\
\hline 3386 & $\mathrm{R}$ & 1963 & $\mathrm{~J} \quad 11$ & & 36.8 & -2.1 & & & C. $\mathrm{G}$ & & & D 16 \\
\hline 3387 & C & 196 & J 15 & & 38.3 & -1.1 & 11 & 4 & $Y A b a$ & 3.111 & & \\
\hline 3388 & $\mathrm{C}$ & 1963 & J 19 & & & -1.1 & 11 & 4 & VI Abanilla & & & $\mathrm{J} 18$ \\
\hline 3389 & c & 1963 & J 25 & 113301 & 38.2 & -0.5 & il & 4.0 & IV S. Pola & $\mathrm{m}$ & & J19 \\
\hline 3390 & MI & 1963 & J 26 & 134706 & 35.9 & -3.8 & 33 & 4.5 & Alboran & & & B 13 \\
\hline & MI & l & $J=2$ & & & 6.0 & 33 & & & & & D08 \\
\hline 3392 & MI & 1963 & J 31 & & & -2.0 & 11 & 3. & & & & E 16 \\
\hline 3393 & $\mathrm{R}$ & 1963 & F 10 & & 38.2 & -0.5 & & & $S .1$ & & (.) & J19 \\
\hline 3394 & MI & 1963 & M 18 & 11 & 39.1 & -0.4 & II & 4 & $\mathrm{Ja}$ & & & $\mathrm{L}, 20$ \\
\hline 3395 & $\mathrm{MI}$ & 196 & M. 28 & 042 & 35.8 & -4.9 & II & 4 & & & & B11 \\
\hline 3396 & $R$ & 1963 & MI 28 & & 38.0 & -1.0 & & & Novelda & 2. & (.) & II 18 \\
\hline 3397 & $\vec{M}$ & 1963 & MI 31 & & 35.8 & -8.9 & 33 & 5.2 & G. Cadiz & & & B03 \\
\hline & $\mathrm{R}$ & 1963 & A 3 & & 38.0 & -0.7 & & & Torrevicja & & $()$. & II 19 \\
\hline 3399 & MI & 1963 & A 21 & & 35.8 & -4.2 & 11 & 4 & & & & B12 \\
\hline 3400 & MI & 1963 & Y 10 & 195940 & 37.1 & -2.8 & 11 & 3.9 & Paterua & & & E15 \\
\hline
\end{tabular}


(Tab. I, cont.)

\begin{tabular}{|c|c|c|c|c|c|c|c|c|c|c|c|c|c|}
\hline Order & $k$ & year & date & $I I$ ( $\mathrm{CT})$ & Lat $\mathrm{N}$ & Longr & h & $m$ & Int. & Localities & \multicolumn{2}{|c|}{ Notes } & Map \\
\hline 3401 & C & 1963 & Y 30 & 015448 & 37.7 & -2.0 & II & 4.7 & & Velez-Rubio & $\mathrm{m}$ & (.) & 1116 \\
\hline 3402 & c & 1963 & $\mathrm{U} 26$ & 102705 & 35.0 & -3.2 & n & 5.0 & III & Melilla & 8. & & B 14 \\
\hline 3403 & $R$ & 1963 & $U 27$ & 114520 & 37.2 & -2.2 & & & IV & Olula & & $()$. & $\mathrm{E} 16$ \\
\hline 3404 & II & 1963 & U 27 & $2243+7$ & $3 \pi .1$ & -3.2 & 11 & 4.1 & & Olula & & & E 16 \\
\hline 3405 & II & 1963 & J, 25 & 014212 & 35.8 & -3.4 & n & 4.2 & & Alboran & & & $\mathrm{B} 14$ \\
\hline 3406 & MI & 1963 & G $\quad 1$ & 014928 & 37.0 & -8.0 & 33 & 4. & & S. Vicente & & & D04 \\
\hline 3407 & II & 1963 & G 2 & 104919 & 35.0 & -8.6 & 33 & 5. & & W.Marruecos & & (.) & A03 \\
\hline 3408 & MI & 1963 & G 19 & 183236 & 38.3 & -8.7 & $n$ & 4.5 & & W. Roca & & & J03 \\
\hline 3409 & MI & 1963 & G 24 & 012420 & 35.7 & -3.6 & $n$ & 4 & & Alboran & & & B 13 \\
\hline 3410 & II & 1963 & $G 24$ & 114141 & 4.28 & +0.1 & 11 & 4.4 & & Pirineos & & & U21 \\
\hline 3411 & II & 1963 & G 30 & 000430 & 36.6 & -2.0 & II & 3.9 & & Almeria & 1. & & $\mathrm{D} 16$ \\
\hline 3412 & II & 1963 & & 004 & 35.6 & -4.0 & II & 4 & & Albo & & & B12 \\
\hline 3413 & II & 1963 & S 11 & 164855 & 36.8 & -3.0 & ll & 4. & & Berja & & & Dl4 \\
\hline 3414 & $\mathrm{R}$ & 1963 & S 13 & 050440 & 41.6 & +1.4 & & & & Urgel & 5. & $()$. & $\mathrm{S} 23$ \\
\hline 3415 & $M I$ & 1963 & S 16 & 145207 & 37.1 & -3.5 & 11 & 3.8 & & S. Nevada & & & E13 \\
\hline 3416 & $\mathrm{MI}$ & 1963 & S 25 & 222205 & 39.2 & +0.2 & 11 & 4.6 & & G. Valencia & & & $\mathrm{L} 21$ \\
\hline 3417 & $R$ & 1963 & S 26 & 220115 & 36.7 & -2.8 & & & & Roquetas & & (.) & $\mathrm{D} 15$ \\
\hline 3418 & MI & 1963 & S 29 & 123150 & 37.4 & -4.1 & n & 4.2 & & Montefrio & & & $\mathrm{E} 12$ \\
\hline 3419 & II & 1963 & 01 & 071558 & 37.7 & -2.5 & il & 4.1 & & Orce & & & $\mathrm{H} 15$ \\
\hline 3420 & MI & 1963 & 06 & 113936 & 37.3 & -4.0 & 11 & 4.1 & & Montefrio & & & $\mathrm{E} 12$ \\
\hline 3421 & II & 1963 & 013 & 230658 & 37.0 & -4.7 & n & 4. & & a & & & D 11 \\
\hline 3422 & MI & 1963 & 015 & 18 & 37.2 & -4.6 & n & 4 & & & & & Eil \\
\hline 3423 & MI & 1963 & O 24 & 03 & 37.0 & -4.6 & n & 4 & & era & & & DIl \\
\hline 3424 & II & 1963 & $N 2$ & 124518 & 35.4 & -4.6 & II & 4 & & Al & & & All \\
\hline 3425 & MI & 1963 & $\mathrm{~N} 18$ & 0 & 35.8 & -2.7 & II & 4 & & $\mathrm{~N}$ & & & 1315 \\
\hline 3426 & MI & 1963 & $N 18$ & 190919 & 38.0 & 0 & ll & 4. & & Medit & & & 1120 \\
\hline 3427 & MI & 1963 & N 19 & & 36.4 & -4.0 & II & 3 & & & & & $\mathrm{Cl} 2$ \\
\hline 3428 & M & 1963 & \25 & 035421 & 37.1 & -3.9 & 11 & 4.2 & & Alha & & & E 13 \\
\hline 3429 & MI & 1963 & N 26 & & 37.0 & -8.0 & $n$ & 4. & & S. $M I$ & & & D04 \\
\hline 3430 & $\mathrm{C}$ & 1964 & J 12 & 212453 & 37.2 & -3.8 & n & 4.7 & IV & Isachar & & & E 13 \\
\hline 34 & MI & 1964 & J 13 & & 38.2 & -9.3 & II & 4 & & ST & & & $\mathrm{J} 02$ \\
\hline 3 & $\mathrm{C}$ & 1964 & J 29 & 51 & 37.3 & -3.7 & n & 4 & VI & P. Puent & 2. & & E13 \\
\hline 3433 & III & 1964 & F' 19 & 02 & 35.7 & -3.3 & n & 4 & & Albor & & & B14 \\
\hline 3434 & C & 1964 & MI 1 & 101959 & 37.8 & -1.5 & II & 4 & III & Aledo & & & $\mathrm{H} 17$ \\
\hline 3435 & II & 1964 & $\mathbf{M} 5$ & & & -3.3 & 11 & 3 & & S. Nevada & & & V:14 \\
\hline 3436 & $\mathbf{B}$ & 1964 & MI 15 & 223026 & 36.0 & -7.7 & 100 & 6.2 & VIII & G. Cadiz & $0 . \mathrm{m}$ & (.) & B05 \\
\hline 3437 & $\mathrm{R}$ & 1964 & II 20 & 063 & 37.7 & -1.8 & & & & Lorea & & & 1117 \\
\hline 3438 & $M I$ & 1964 & A 2 & 160231 & 38.2 & -1.1 & n1 & 4. & & Abanilla & & & J 18 \\
\hline 3439 & MI & 1964 & & & 37 & -4.7 & 11 & 4 & & dilla & & & D 11 \\
\hline 3440 & $\mathrm{C}$ & 1964 & A 3 & 122833 & 38.2 & -1.1 & 11 & 4 & $\mathrm{~V}$ & Abanilla & & & $\mathrm{J} 18$ \\
\hline 3441 & MI & 1964 & A 9 & & 3 & -4.3 & 11 & 4 & & A & & & B 12 \\
\hline 344 & MI & 1964 & $\Lambda 11$ & & & -1.0 & li & & & & & & $\mathrm{J} 18$ \\
\hline 3443 & MI & 1964 & A 14 & 102 & 38.2 & -1.0 & 11 & 4 & & $A$ & & & \\
\hline 3444 & MI & 1964 & 115 & & 38.4 & -1.8 & II & 4 & & & & & $\mathrm{~J} 17$ \\
\hline 3445 & $\mathrm{C}$ & 1964 & $\Lambda 17$ & 185 & 37.2 & -3.7 & 11 & 4 & II & S. Fe & & & E 13 \\
\hline 3446 & WI & 1964 & $\Lambda 21$ & & 36 & +3.3 & II & 4 & & & & & $\mathrm{C} 27$ \\
\hline 3447 & B & 1964 & A 26 & & 36.2 & -4.6 & 65 & 4 & & & & & B 11 \\
\hline 3448 & II & 1964 & A 26 & 202851 & 36.3 & -4.1 & 11 & 4.3 & & Mediterran. & & & $\mathrm{Cl2}$ \\
\hline
\end{tabular}


(Tab. I, cont.)

\begin{tabular}{|c|c|c|c|c|c|c|c|c|c|c|c|c|c|}
\hline Order & $k$ & year & date & $I I\left(\mathrm{U}^{\prime} \mathrm{T}\right)$ & Lat $N$ & Longr & li & $m$ & Int & Localities & \multicolumn{2}{|c|}{ Notes } & Map \\
\hline $\begin{array}{l}3449 \\
3450\end{array}$ & $\begin{array}{l}\mathrm{MI} \\
\mathrm{MI}\end{array}$ & $\begin{array}{l}1964 \\
1964\end{array}$ & $\begin{array}{l}\text { A } 27 \\
\text { A } 30\end{array}$ & $\begin{array}{l}023441 \\
010357\end{array}$ & $\begin{array}{l}36.5 \\
37.0\end{array}$ & $\begin{array}{l}-1.5 \\
-4.8\end{array}$ & $\begin{array}{l}\text { n } \\
\text { n }\end{array}$ & $\begin{array}{l}4.4 \\
4.2\end{array}$ & & $\begin{array}{l}\text { Mediterran. } \\
\text { Bobadilla }\end{array}$ & 2. & & $\begin{array}{l}\text { Cl7 } \\
\text { D11 }\end{array}$ \\
\hline 3451 & $\mathrm{AI}$ & 1964 & $\mathrm{Y}$ & 233002 & 37.5 & -2.1 & $n$ & 4.2 & & Albox & & & E16 \\
\hline 3452 & MI & 1964 & $\overline{\mathrm{Y}} 9$ & & 38.2 & -1.0 & $n$ & 4 & & banilla & & & 18 \\
\hline 3453 & $M$ & 1964 & Y 13 & 134621 & 35.8 & -1.7 & n & 4 & & Mediterran. & & (.) & 1317 \\
\hline 3454 & 13 & 1964 & Y 13 & 175 & 35.8 & -5.0 & $9 \tilde{5}$ & t. & & Alboran & & & 310 \\
\hline 3455 & B & 1964 & U 9 & 023332 & 37.8 & -2.5 & 5 & 5.2 & VII & Il uescar & 13. $\mathrm{ml}$ & $()$. & II 15 \\
\hline 3456 & C & 1964 & U 11 & 225014 & 37.8 & -2.6 & n & 4.3 & III & Galera & & & 1115 \\
\hline $3+57$ & $M$ & 1964 & U 12 & 093336 & 37.7 & -2.5 & n & 3. & & Orce & & $()$. & 1115 \\
\hline 3458 & $M$ & 1964 & U 13 & 160048 & 37.7 & -2.3 & $\mathrm{n}$ & 4.0 & & Orce & & & 1116 \\
\hline 3459 & $M$ & 1964 & U 18 & 112610 & 37.5 & -3.6 & $n$ & 3 & & Campotejar & 1. & & E13 \\
\hline 3460 & $\mathrm{M}$ & 1964 & U 21 & 024405 & 37.8 & -3.6 & $n$ & 3.8 & & Rea & & & 113 \\
\hline & $\mathrm{M}$ & 1964 & U 26 & & 37.3 & -2.2 & $\mathrm{n}$ & 4. & & & 1. & & 16 \\
\hline 3462 & MI & 1964 & U 27 & 002 & 37.8 & -2.5 & $\mathrm{n}$ & 4 & & & 3. & $()$. & 115 \\
\hline 3463 & MI & 1964 & U 29 & 00 & 37.8 & -2.5 & n & 4 & & & & & 115 \\
\hline 3464 & $\mathrm{C}$ & 1964 & L 3 & 213531 & 37.9 & -1.5 & n & 4.0 & IV & Pliego & $\mathrm{m}$ & & 1117 \\
\hline 3465 & $\mathrm{C}$ & 1964 & L. 13 & 122 & 37.1 & -2.0 & n & 4 & $\mathrm{~V}$ & & & (.) & 16 \\
\hline 3466 & MI & 1964 & I. 15 & 094 & 35.5 & +4.3 & 33 & 4. & & Setif & & $()$. & A29 \\
\hline 3467 & $\mathbf{R}$ & 1964 & L. 15 & 110 & 35.3 & -0.3 & & & & & (BCIS) & & $\wedge 20$ \\
\hline 3468 & MI & 1964 & L 19 & 020707 & 37.2 & -5.5 & n & 3.0 & & MLoron & & & L09 \\
\hline 3469 & M & 1964 & I 28 & 220942 & 42.7 & -1.5 & n & 4. & & Pamplona & & & U17 \\
\hline $3 \pm 70$ & $\mathbf{R}$ & 1964 & G 15 & 064520 & 36.7 & -4.3 & & & & Malaga & 3. & (.) & $\mathrm{D} 12$ \\
\hline 3471 & $M$ & 1964 & G. 17 & & 37 & -2.8 & $n$ & 4. & & & & & 1115 \\
\hline $3 \pm 72$ & $\mathrm{C}$ & 1964 & G 29 & 0 & 4 & +0.1 & n & 4. & VI & Bat & & (.) & J 21 \\
\hline 3473 & MI & 1964 & & & 3 & -5.3 & II & 3 & & les & & & D10 \\
\hline 3474 & C & 1964 & & 3 & 3 & -4.4 & n & 4 & IV & ermeja & 21. & & 12 \\
\hline 3475 & $\mathrm{C}$ & 1964 & & & 37. & $-\cdots 3.5$ & 15 & 4 & VI & & 21. & (.) & $\mathrm{D} 13$ \\
\hline $3+76$ & $\mathrm{C}$ & 1964 & & & 36 & -4.4 & n & 4 & IV & eja & 4. & $()$. & D 12 \\
\hline 3477 & $\mathrm{R}$ & 1964 & S 11 & & 36 & -4.7 & & & III & Abdalagis & 3. & & D11 \\
\hline 3478 & $\mathrm{R}$ & 1964 & S 20 & 092030 & 36.7 & -4.3 & & & III & Malaga & & (.) & D 12 \\
\hline $3+79$ & $M$ & 1964 & S 29 & 051928 & 37.4 & -2.0 & n & 3.9 & & II uereal & & & 18 16 \\
\hline 3480 & $M$ & 1964 & $\begin{array}{ll}0 & 2\end{array}$ & & 35.6 & 0.1 & $\mathrm{n}$ & 4 & & Perregaux & & & 21 \\
\hline & MI & 4 & & & & -4.6 & $n$ & & & & & & 11 \\
\hline 82 & M & 1964 & $\begin{array}{ll}0 & 10\end{array}$ & & 37. & -2.7 & $n$ & & & $A$ & & & E 15 \\
\hline & $M$ & 1964 & () 17 & & & $-2,5$ & II & 3 & & & & & II 15 \\
\hline 84 & $\mathrm{E}$ & 1964 & 021 & & 36 & $-4-4.5$ & 33 & & VII & & (BC & (.) & $\mathrm{C} 29$ \\
\hline & $\mathrm{C}$ & 1964 & $\mathrm{~N} 2$ & & 36 & -2.6 & $n$ & 4 & III & eria & & & D) 15 \\
\hline 3486 & $\mathrm{MI}$ & a 964 & & & 37. & -2.3 & nl & & & & & & 1116 \\
\hline & $\mathrm{R}$ & 1964 & $\mathrm{~N} \quad 9$ & & 36.6 & -9.6 & & & & & (BCIS) & (.) & \\
\hline 88 & MI & 1964 & N 23 & & 36.9 & -4.4 & In & 4. & & $\mathrm{Ca}$ & & (.) & D 12 \\
\hline 3489 & $\mathrm{C}$ & 1964 & N 26 & 231459 & 38.8 & -0.4 & Il & 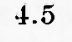 & V & $\mathrm{Ca}$ & $\mathrm{m}$ & $()$. & $\mathrm{K} 20$ \\
\hline 3490 & $M I$ & 196 & D 21 & 000459 & 39.4 & -0.2 & $n$ & & & & & & $\mathrm{~L} 20$ \\
\hline & $\mathrm{MI}$ & 196 & D 23 & & 36 & -4.3 & $n$ & & & & 14 & & \\
\hline & $\mathrm{MI}$ & 1964 & D 29 & & 37.2 & -4.2 & $\mathrm{n}$ & & & & I & & 2 \\
\hline & M & 1965 & $\begin{array}{ll}\mathrm{J} & \mathrm{l}\end{array}$ & & 35.4 & +4.5 & $n$ & & & & & & A29 \\
\hline 94 & $\mathrm{C}$ & 1965 & & 21 & 35 & +4.5 & n & 5. & VIII & M'sila & 2. & (.) & A 29 \\
\hline 3495 & $M$ & 1965 & J $\quad 9$ & & 36.5 & -4.3 & $\mathrm{n}$ & $t$. & & S. Malaga & & & C12 \\
\hline 3496 & $M$ & 1965 & J 25 & 165309 & 36.7 & -2.0 & n & 9.5 & & C. Gata & & & $\mathrm{D} 16$ \\
\hline
\end{tabular}


('Tab. I, cont.)

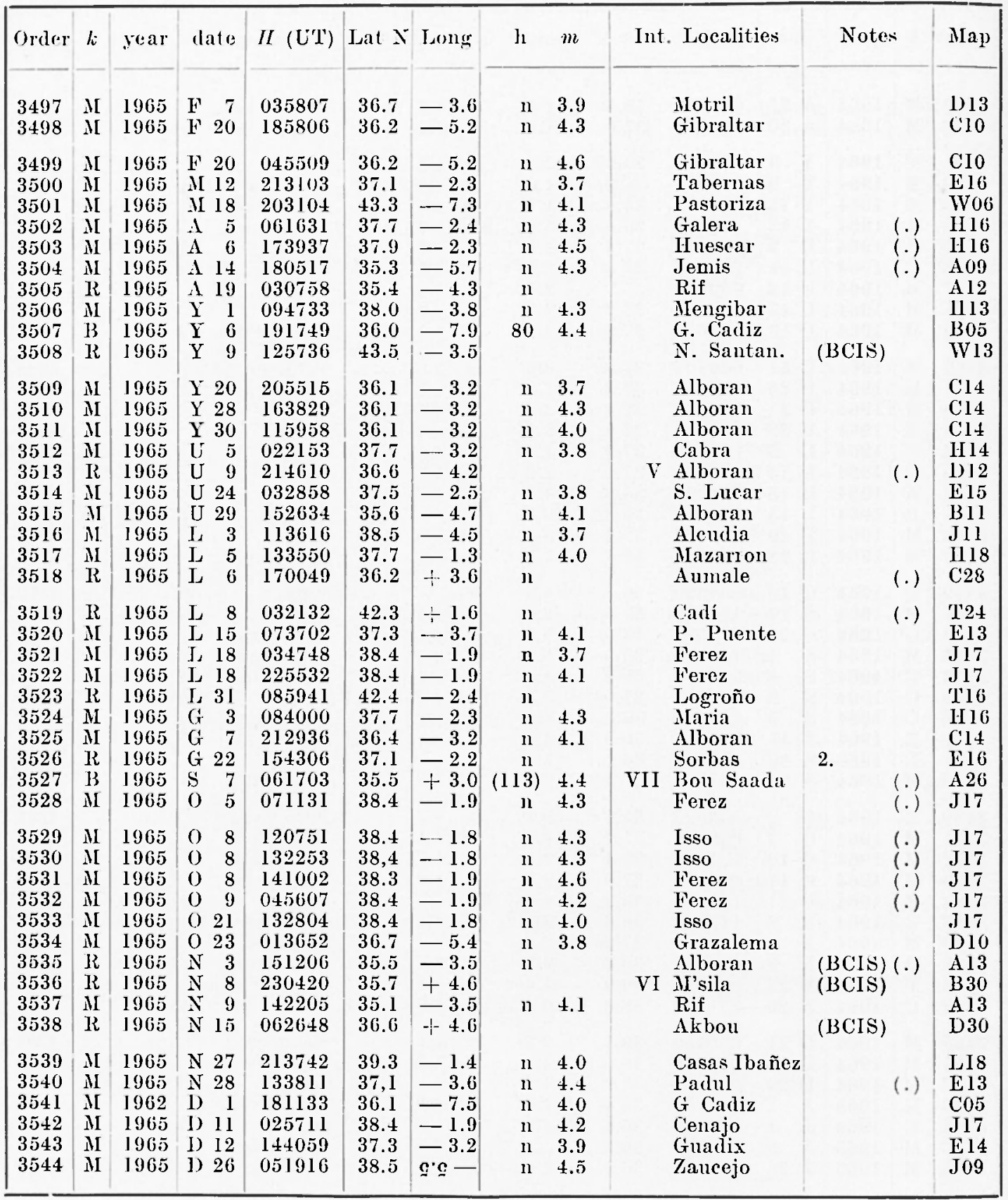


Table II - Notes QuOTED IN Table 1.

Order.

$\begin{array}{lll}3311 . & \text { BCIS } 41.5-6.3 \\ 3312 . & \text { BCIS } 36.2--6.8 \\ 3313 . & \text { BCIS } 38.3-6 & -3.3 \\ 3321 . & \text { BCIS } 37.3--3.8 \\ 3324 . & \text { BCIS } 41.8-2.5 \\ 3326 . & \text { Lisboa } 36.5--8.7 \\ 3327 . & \text { BCIS } 36.5+1.2\end{array}$

3346. Alicante $35.9-3.6$, BCIS $37.3-4.0$, USCGS $37.3-4.9$ $h=25$, LCIS $h=n$

3347. conjectural

3350. BCIS $35.3--9.8$

3356. BCIS $43.6--5.3$

3360. BCIS $36.3--4.3$

3364. conjectural

3366. USCGS $36.7+1.6, h=33$, LCSS $h=33$

3367. conjectural

3368. USCGS $36.5+1.6 h=15, \quad$ LCSS $h=33$

3370. USCGS $36.5-9.0 h=33$, LCSS $h=33$

3380. conjectural

3384. conjectural

3385. BCIS $42.0+0.5$

3386. conjectural

3393. conjectural

3396. conjectural

3397. USCGS $35.1-9.3 h=33$, $\operatorname{BCIS} 35.5-8.2$

3398. conjectural

3401. BCIS $37.7-2.5$

3402. BCIS $36.0-4.2$, USCGS $35.3-3.7 h=33 m=4.6$

3403. conjectural

3407. USCGS $34 . \prime-8.9 h=33$.

3414. conjectural

3417. conjectural

3436. BCIS $36.0-7.5$, USCGS $36.2-7.6 \mathrm{~h}=27 \mathrm{~m}=6.2$

3437. conjectural

3453. BCIS $35.4-1.9$

3454. BCIS $36.4-5.1$

3455. BCIS $37.6-2.4$

3456. BCIS $37.7-2.6$

3457. BCIS $37.7-2.6$

3462. BCIS $37.6-2.5$ 
('liable: II, cont.)

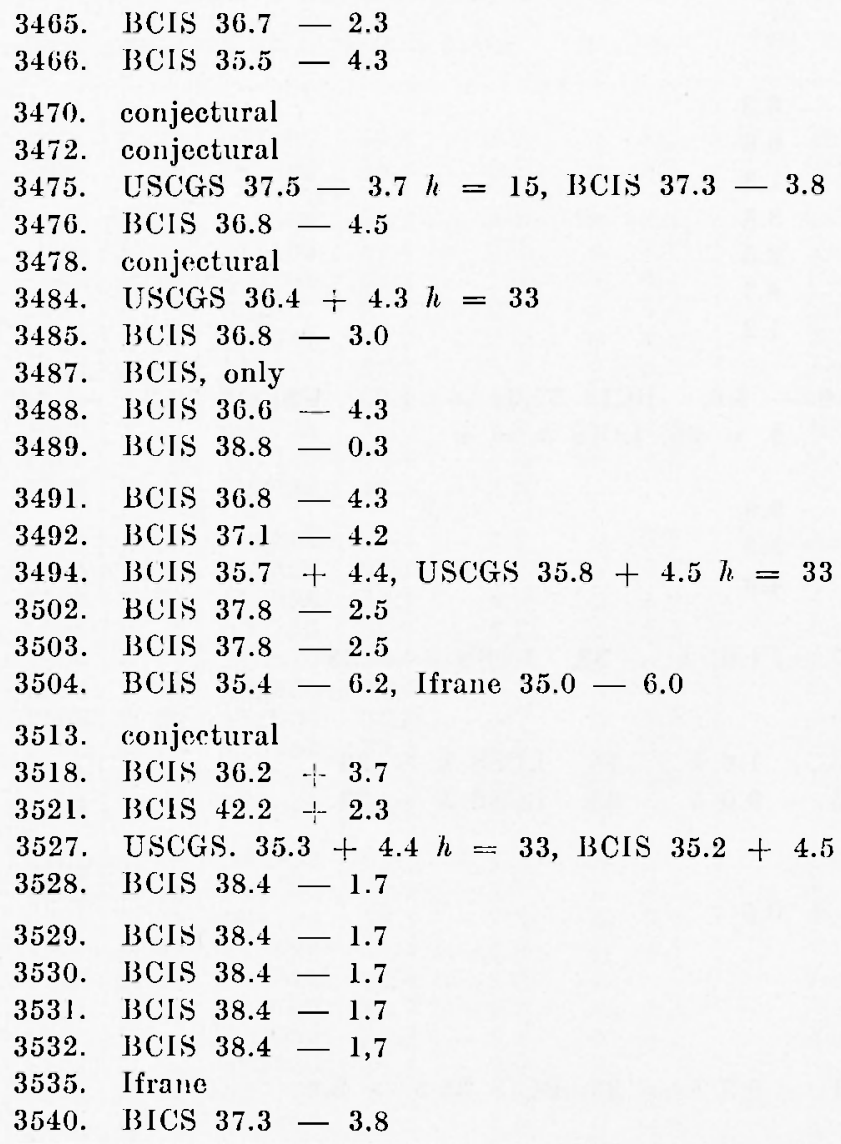

by means of the computer program EPICO. We plan to apply the same method to every shooks with recording data in six or more stations.

By quality, it is possible to take three groups of shocks, regarding its epicentral location:

ronjectural $R j$. . . . . . . . . . . . . . . . 13 shocks

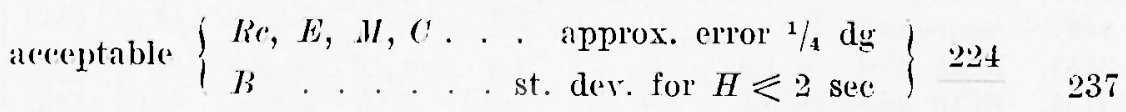

lixception made for $B$ kind, the error quota is unknown in the epicentral location as a consequence the uncertain focal depth. The preliminary epicentral determinations have been made in the LCSS by J. L. Flores-Calderón, who applied systematically a graphic method 
on a map scale 1:2.000.000, using values deduced from the Polumb and Evison travel-time tables. Magnitude for the $I, C, B$ shocks has been computed by means the formula explained in CM, using body waves, which is

$$
m=0.63 \log A / T+1.207 \log 11^{\circ}+4.175+d m .
$$

Recently, in MA, a new formula was deduced to compute $m$ from $\mathrm{S}$ waves and periods close and higher than 0.8 sec. Differences between these last values and those obtained from [1] are lower than $1 / 3$ unities of magnitude, approximately. Wherefore, it is acceptable now to group shocks of $1 / 4 m$ in order to make statistical studies.

\section{SEISHIC ACTIVE QUadRICles.}

The area was divided (see SD) with a net of parallels and meridians of $1 / 2$ geographical degrees, identifying everyone by means an alphameric code: a letter to latitude and two cipher's to longitude, respectively. Table III lists the seismic active quadricles and the chronological ordinal number for the corresponding located shocks. Migration was advised during prior studies; even, secondary maximum for 1801-1960 becames primary for 1961-1965 as it was suggested in EF, table 18. The migration, for both maxima, is summarized as follows:

\begin{tabular}{|c|c|c|c|c|}
\hline yeats: & $601-1300$ & $1301-1800$ & $1801-1960$ & $196 \mathrm{~L}-1965$ \\
\hline$p^{r i}$ & E 11 & $\mathrm{~K} 02$ & U21 & E13 \\
\hline secoondany: & KO2 & $\mathrm{U} 21 \uparrow$ & E13 & J17-W16 \\
\hline
\end{tabular}

Absolute density, or pereentage of total located shocks, is quoted in Table III, which also shows the relative density corresponding to each region (*). The following (quadricles show major density:

\begin{tabular}{lcc} 
& absolute & per $10^{3} \mathrm{sq} . \mathrm{km}$ \\
\cline { 2 - 2 } $\mathrm{C} 14$ & 3.75 & 1.5 \\
$\mathrm{I} 12$ & 3.35 & 1.3 \\
$\mathrm{E} 13$ & 7.12 & 2.8 \\
$\mathrm{E} 16$ & 4.18 & 1.7 \\
$\mathrm{H} 15$ & 3.35 & 1.3 \\
$\mathrm{~J} 17$ & $1.1 \mathrm{~S}$ & 1.7 \\
$\mathrm{~J} 1 \mathrm{~S}$ & 3.35 & 1.3
\end{tabular}

(*) Relative densit, is the ratio between absolute density and surface, expressed in percentage of the total area (see $\mathrm{EF}$ ). 
'Table III - SEISMIC ACTIVE QUADRICLES.

\begin{tabular}{|c|c|c|c|c|c|c|}
\hline \multicolumn{2}{|c|}{ Z Reg. } & Quad & $\begin{array}{l}\text { Stock ordina! number } \\
\text { (see SD, EF) }\end{array}$ & A.D. MRD & Energy & $\varnothing$ \\
\hline \multirow[t]{6}{*}{$A$} & 1 & $\begin{array}{l}1106 \\
\times 06\end{array}$ & $\begin{array}{l}3501 \\
3322-3356\end{array}$ & $\begin{array}{ll}0.4 & \\
0.8 & 0.2\end{array}$ & $\begin{array}{ll}0.1 & (18) \\
0.1 & (20)\end{array}$ & $\begin{array}{l}7.4 \\
8.4\end{array}$ \\
\hline & 2 & W11 & 3314 & $\begin{array}{l}\text { average } \\
0.4\end{array}$ & $2.02(18)$ & . \\
\hline & & W13 & 3508 & $0.4 \quad 0.3$ & . & . \\
\hline & $4 a$ & $\mathrm{~L} 16$ & 3373 & 0.4 & $0.1(17)$ & 7.0 \\
\hline & & $\mathrm{L}_{S} 17$ & $\begin{array}{l}(3347) \\
3311-3339\end{array}$ & 0.4 & $0.100)$ & 81 \\
\hline & & S15 & 3324 & $\begin{array}{l}0.8 \\
0.4 \quad 1.4 \\
\text { average }\end{array}$ & $\begin{array}{l}0.1(19) \\
2.20(18)\end{array}$ & 8.0 \\
\hline \multirow[t]{7}{*}{$B$} & 3 & T1 6 & 3523 & 0.4 & & \\
\hline & & U17 & 3469 & $\begin{array}{l}0.4 \quad 0.4 \\
\text { average }\end{array}$ & $\begin{array}{l}0.1(17) \\
2.00(15)\end{array}$ & 7.0 \\
\hline & 4 & U21 & $3410-3 \pm 72$ & $\begin{array}{l}0.4 \quad 0.3 \\
\text { average }\end{array}$ & $\begin{array}{l}0.1(19) \\
2.00(1 \%)\end{array}$ & 8.0 \\
\hline & $9 b$ & $\mathrm{~S} 22$ & $3361-3385$ & $\begin{array}{l}0.8 \quad 0.2 \\
\text { average }\end{array}$ & $\begin{array}{l}0.3(19) \\
6.00(17)\end{array}$ & 8.1 \\
\hline & 10 & $\begin{array}{l}\$ 23 \\
\$ 24\end{array}$ & $\begin{array}{l}(3414) \\
3519\end{array}$ & 0.8 & & . \\
\hline & & T25 & $\begin{array}{l}3019 \\
3381\end{array}$ & $\begin{array}{l}0.4 \\
0.4\end{array}$ & $0.1(20)$ & 8.4 \\
\hline & & 'T26 & 3355 & $\begin{array}{l}0.4 \quad 0.5 \\
\text { average }\end{array}$ & $2.00(19)$ & \\
\hline \multirow[t]{8}{*}{$\mathrm{C}$} & 11 & J 02 & 3431 & 0.4 & 0.1 (18) & 7.4 \\
\hline & & $J 03$ & 3408 & $\begin{array}{l}0.4 \quad 0.3 \\
\text { average }\end{array}$ & $\begin{array}{l}0.1(18) \\
4.00(16)\end{array}$ & 7.4 \\
\hline & 12 & J 09 & 3544 & $0.4 \quad 0.2$ & 0.1 (18) & 7.4 \\
\hline & 13 & Dol & 3487 & $\begin{array}{l}\text { average } \\
0.4\end{array}$ & $2.00(16)$ & \\
\hline & & Do2 & $3325-3370$ & 0.8 & $0.3(19)$ & 8.1 \\
\hline & & $1) 04$ & $3406-3 \pm 29$ & & $0.3(19)$ & 8.1 \\
\hline & & 1102 & 3318 & $\begin{array}{l}0.4 \quad 1.1 \\
\text { average }\end{array}$ & $1.20(18)$ & . \\
\hline & $14 a$ & D08 & $(3364)-3354-3391-3310$ & $\begin{array}{l}1.7(1.1) \\
\text { average }\end{array}$ & $\begin{array}{l}0.1(19) \\
2.00(1 \gamma)\end{array}$ & 8.0 \\
\hline \multirow[t]{6}{*}{ D) } & $14 b$ & $\mathrm{E} 09$ & $3348-3468$ & $0.8(1.1)$ & $0.2(18)$ & 7.5 \\
\hline & 15 & D 10 & $3320-3473-3534$ & 0.8 & $\begin{array}{l}7.00(10) \\
0.2(18)\end{array}$ & 7.4 \\
\hline & & 1011 & $\begin{array}{l}3421-3423-3439-3450- \\
\quad 3 \pm 77\end{array}$ & 2.1 & $0.2(17)$ & 7.1 \\
\hline & & D 12 & $\begin{array}{c}3399-(3470)-3474-3476- \\
(3478)-3 \pm 91-(3513)\end{array}$ & 3.3 & $0.8(18)$ & 7.9 \\
\hline & & D 13 & $\begin{array}{l}3308-3337-3359-3475- \\
\quad 3498\end{array}$ & 2.5 & $0.7(18)$ & 7.8 \\
\hline & & $\begin{array}{l}\text { D } 14 \\
\text { D } 15\end{array}$ & $\begin{array}{l}3375-3413 \\
3372-3417-3485\end{array}$ & $\begin{array}{l}0.8 \\
1.3\end{array}$ & $\begin{array}{l}0.8(16) \\
0.7(16)\end{array}$ & $\begin{array}{l}6.9 \\
6.8\end{array}$ \\
\hline
\end{tabular}


(Table 1II, cont.)

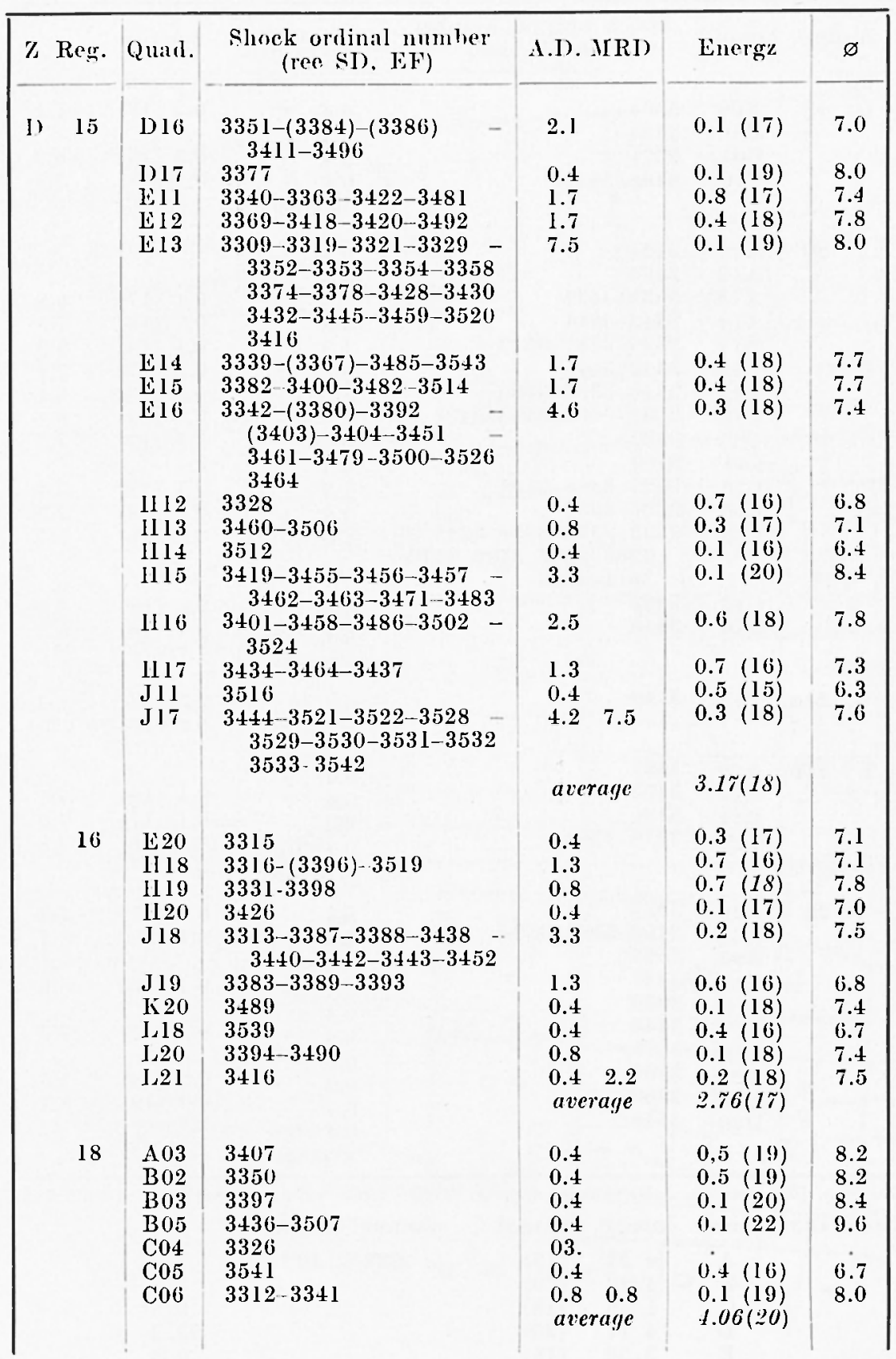


('Table III, cont.)

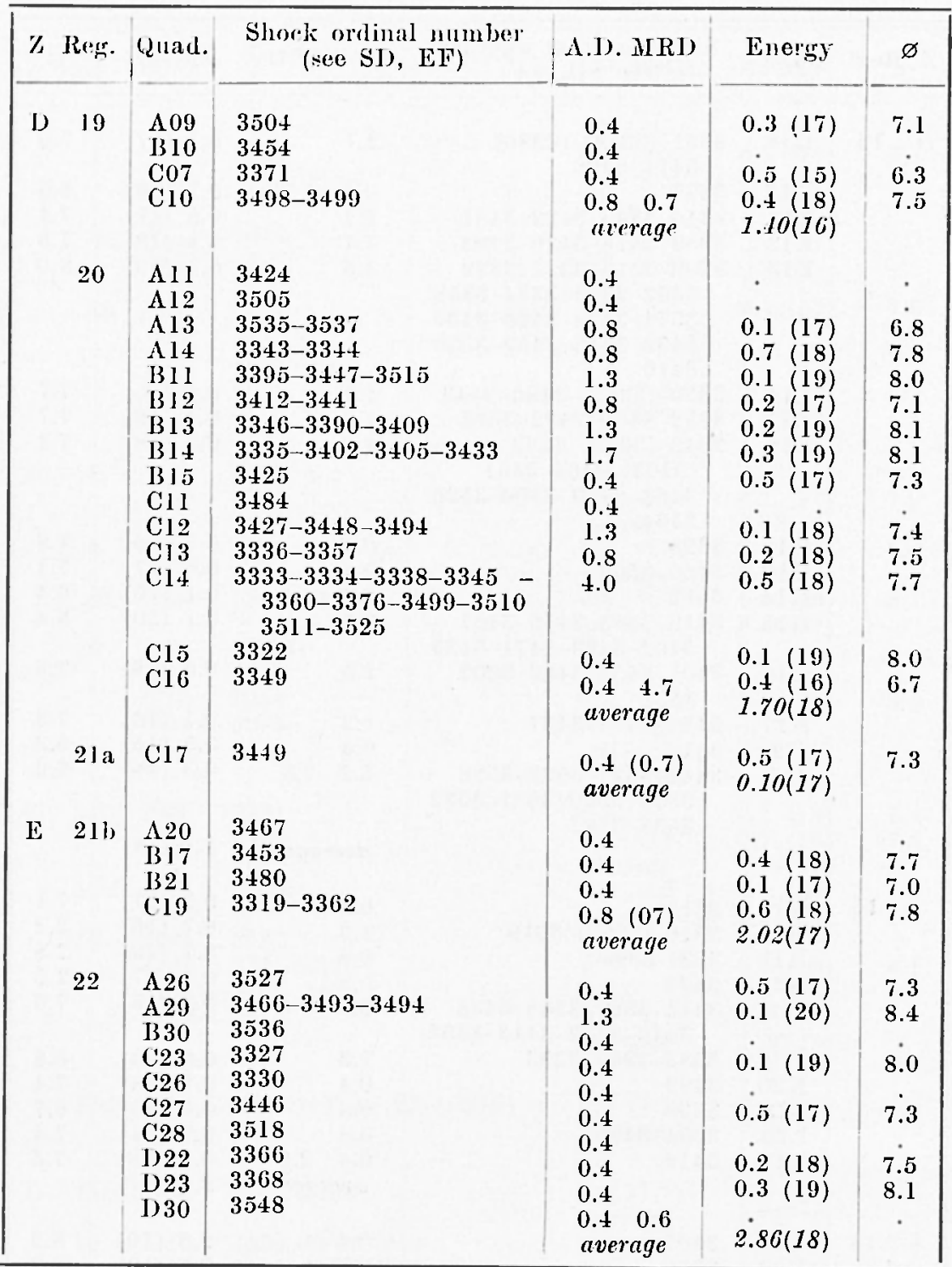

$Z=z o n e$, Reg. = region, A.D. = annual density, MRD =mean regional density, $\varnothing=$ tecnic $\downarrow$ tux. SUMMary: Zone energy released (annual average) \%

$\begin{array}{llllr}\text { A } & 4.22 & (18) & =4.22 \times 10^{18} \mathrm{ergs} & 1.0 \\ \mathrm{~B} & 4.22 & (19) & 4.7 \\ \mathrm{C} & 1.46 & (18) & 0.3 \\ \mathrm{D} & 4.11 & (20) & & 93.2 \\ \mathrm{E} & 3.06 & (18) & 0.8\end{array}$

annual energy $4.41 \times 10^{20}$ ergs (whole area) 100.0 


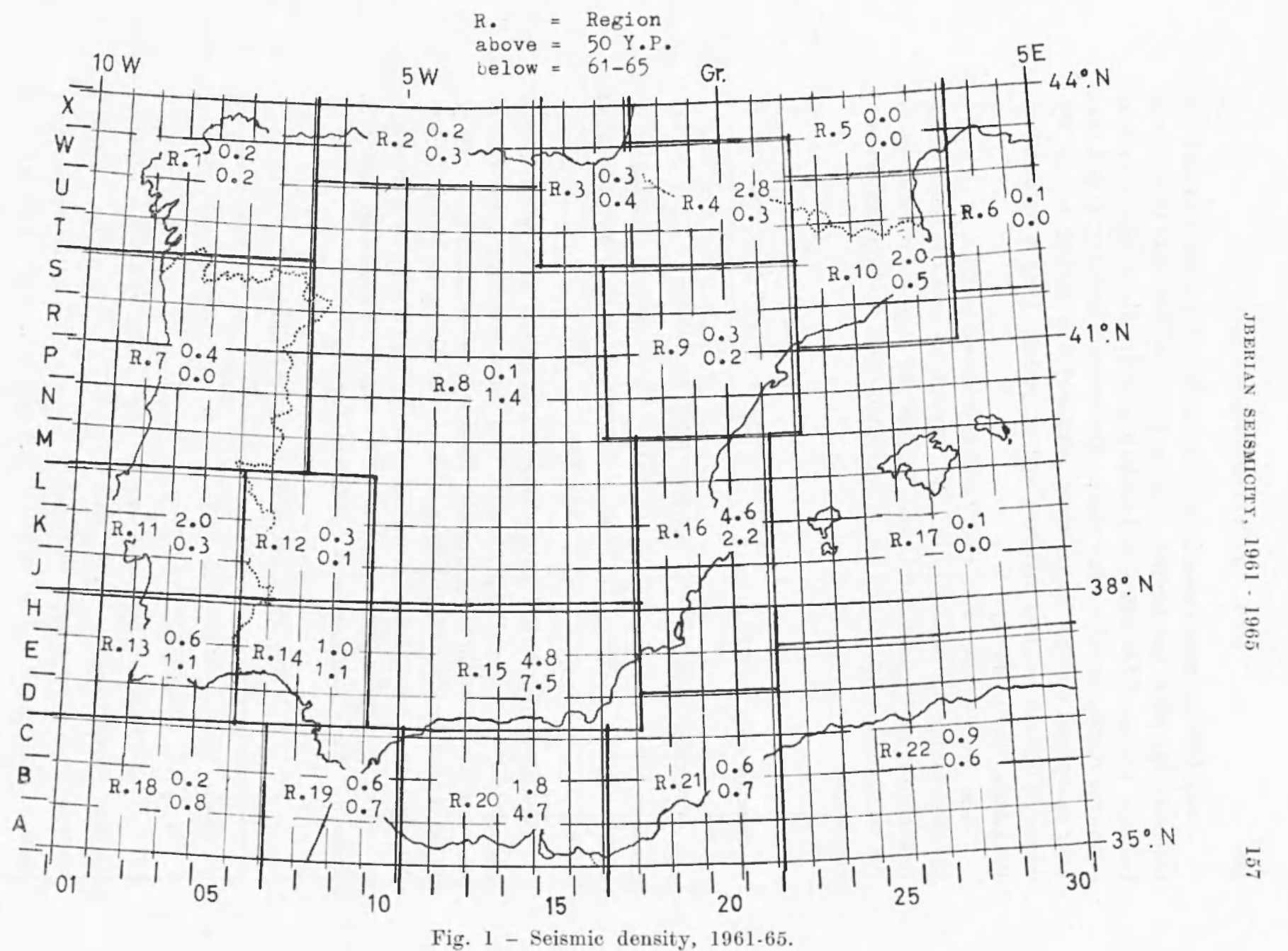


Only E13 was noted as secondary in the 50 YP (values 5.89 and 2.3, respectively) while the highest density was J19, with 8.32 for the said reduced period. This different distribution of density is significative as a general displacement on the area. The seismic activity, in old time very sharpened on the Tagus river (estuary) was moved to the $\mathrm{Py}$ renees Mountain (central massive) and at present time is on Southem Peninsula, like Easterly.

The relative density for every region is shown in Fig 1, which adds the density for the period up to 1960 . It can be seen the positive differences for the regions So. 15 and 20, and the negative differences for the regions No. 11, 4, 10 and 16, in agreement with the comments about migration.

\section{SHOCKS AND DEPTH.}

Regarding depth, shocks are groupped as follows:

shallow . . . . . . $h<50 \mathrm{~km} \mathrm{.} \mathrm{.} \mathrm{.} \mathrm{(isostatic} \mathrm{level)}$ intermediate . . . . . $50<h<413 \mathrm{~km}$. (Byerly discontinuity) drep. . . . . . . . . $h>413 \mathrm{~km}$. . .

The intermediate an the sole deep shocks have bern plotted on a sketch of the area as Fig 2 shows. The shock No 3527 is considered doubtful and refused to be plotted, bicause was deduced $\hbar=160$ by LCSS, $\mathrm{h}=33$ by USCGS and deeper than $113 \mathrm{~km}$ processing EPICO. The epicenter for these intermediate shocks agree with the line suggested by means seismicity, drawing the Alpine Geosyncline (see ES). Table IV sum- marizes shocks groupped by depth.

Table IV - LOCATED SHOCKS BY DEPTII.

\begin{tabular}{|c|c|c|c|c|c|c|}
\hline $\mathrm{h}(\mathrm{km})$ & kind of shoks & $\mathrm{N}$ & $\%$ & $m$ & 5-yard period & level \\
\hline 0 to 5 & very shallow & 18 & 7.6 & 4.2 & $4.5(m) \quad 2.3(\%)$ & surface \\
\hline up to 21 & shallow (normal h.) & 198 & 83.5 & $"$ & 89.4 & Conrad \\
\hline up to 50 & remainder sh. sh. & 13 & 5.4 & $"$ & 7.0 & isostasy \\
\hline up to 413 & intermediate & 8 & 3.4 & $4.4 \sim 6.0$ & $4.5 \sim 5.5$ & Byerly \\
\hline 414 or more & deep & - & 一 & & (only one) & \\
\hline
\end{tabular}




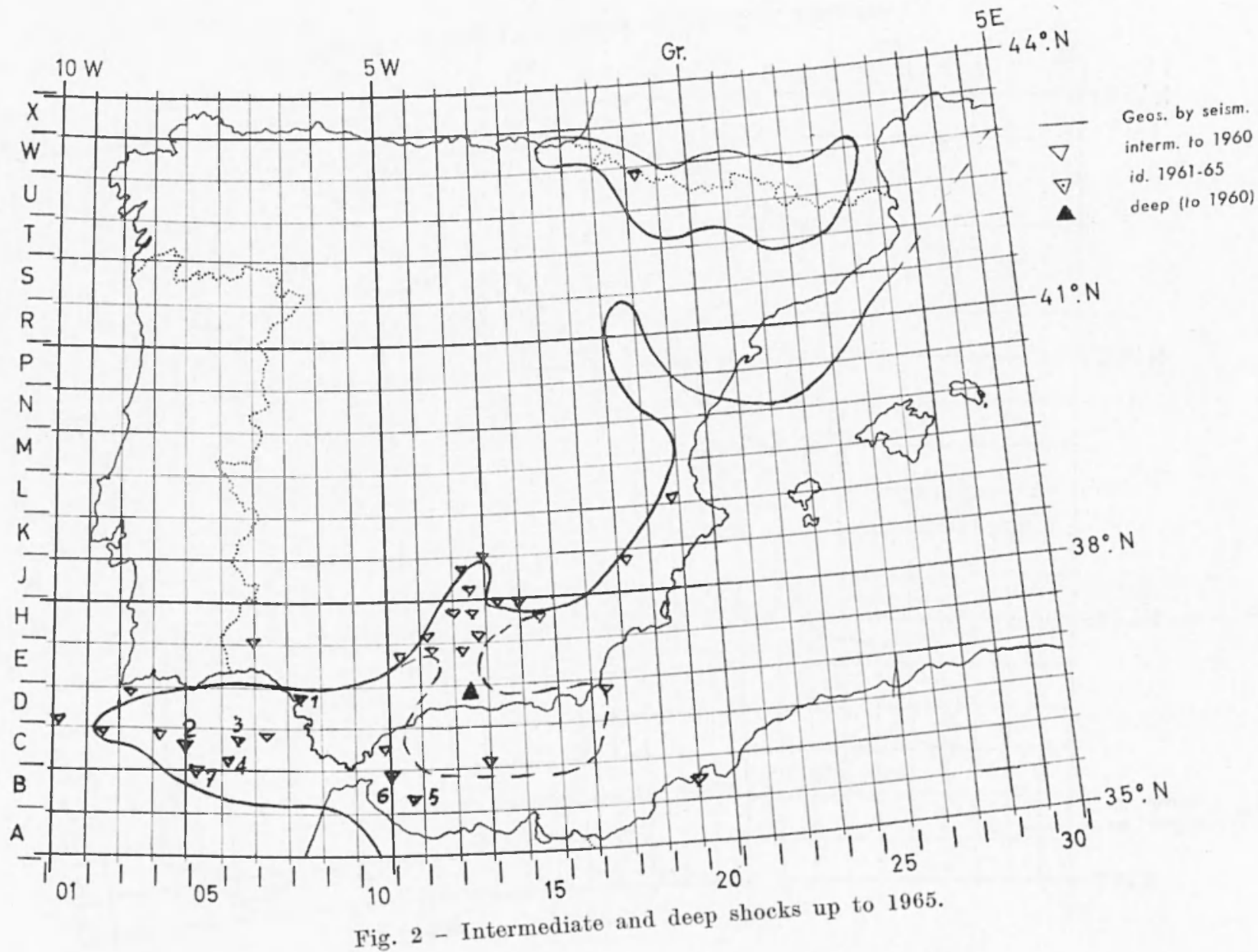

惑 


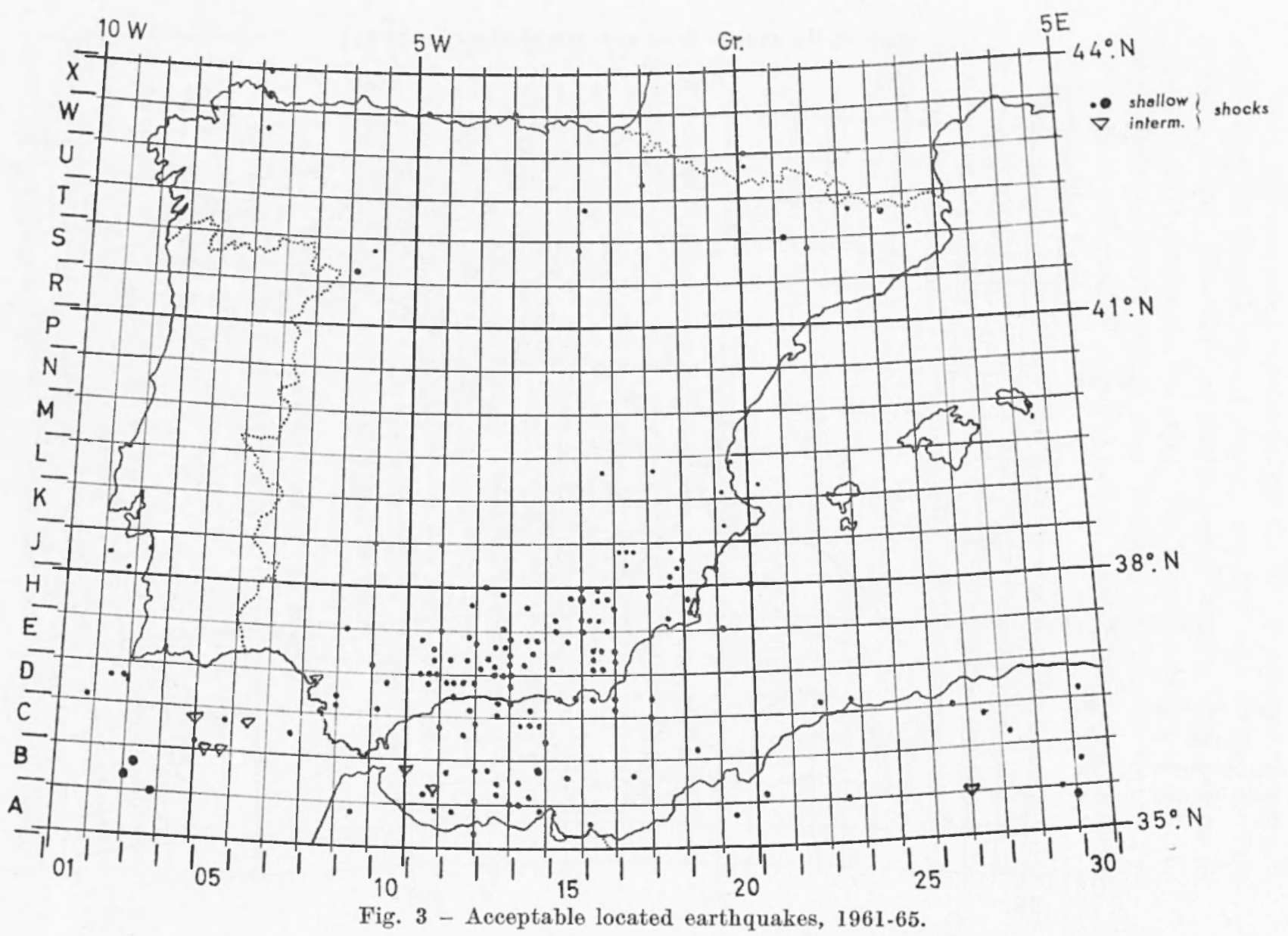




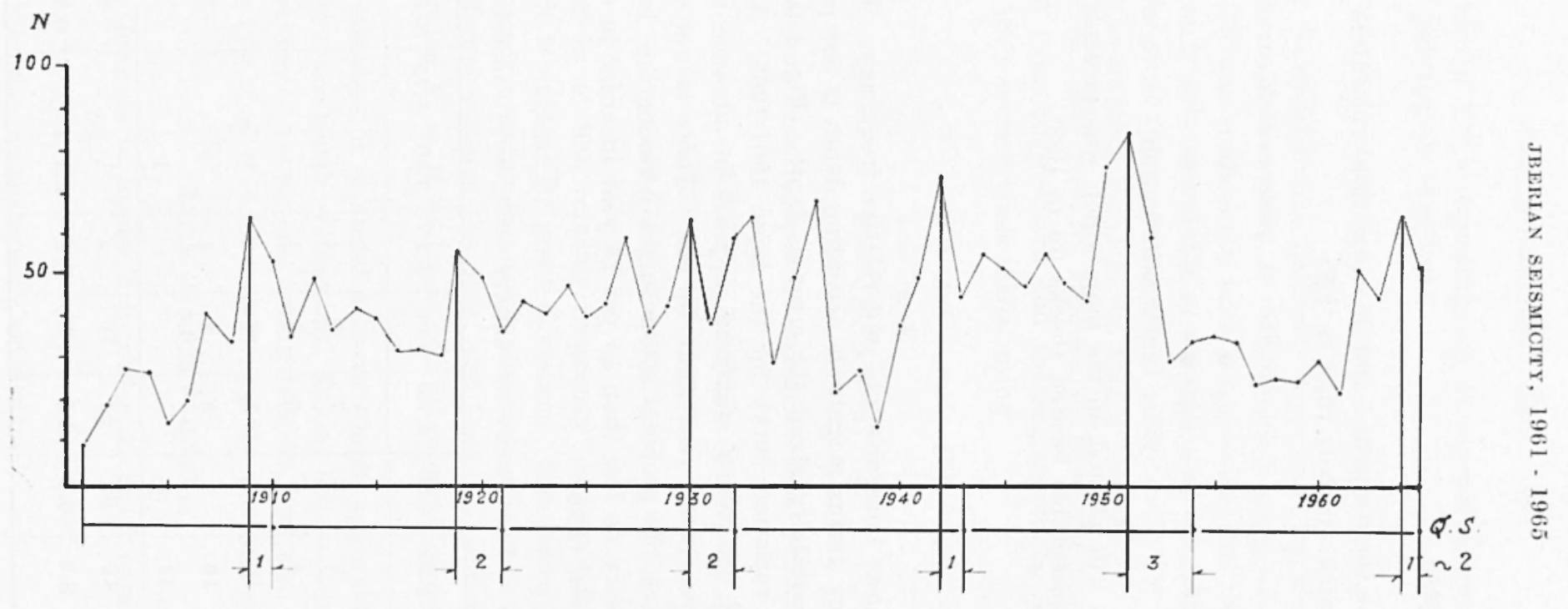

Fig. 4 - Frequency secular variation, 1901-1965. 
כ. EPICliNter MaP.

Fig. 3 shows the computed and no conjectural epicenters for 1961 65, using the same symbols thim in EF:

a) $3<m<5$

b) $5<m<6.5$

c) $m>6.5$ intermediate deep shallow $\nabla$ $\nabla$

(there is not information)

These data will plotted on the map which was presented in EF, built with the acceptable located shocks up to 1960 .

6. Fraquancy.

Table V shows both absolute and relative frequency. The comparison frequency versus magnitude according depth is not presented, because the uncertainity about the error in depth. Fig. 4 is the frequency secular variation curve for the lapse 1901-1965. As it was discussed in EF, frequency maximum appears in advance regalrding the quict-solar-year, one year more or less. There is not sufficient material to attack the problem computing a periodogram, because it should be necessary no less than 15 per 13 year in order to deduce a possible correlation ratio.

Table V - Frequency By groups of $1 / 4 m$ (4. to 6.$)$.

\begin{tabular}{|c|r|r|r|r|r|r|r|r|r|r|}
\hline year & 4.00 & 4.25 & 4.50 & 4.75 & 5.00 & 5.25 & 5.50 & 5.75 & 6.00 & sum \\
\cline { 2 - 10 } 1961 & 4 & 2 & 3 & 7 & 5 & 1 & & & & 22 \\
1962 & 6 & 4 & 9 & 6 & 6 & 1 & & & & 32 \\
1963 & 12 & 10 & 7 & 3 & 3 & 1 & & & & 36 \\
1964 & 18 & 16 & 7 & 8 & 2 & 1 & & & 1 & 53 \\
1965 & 14 & 11 & 8 & & & & 1 & & & 34 \\
\hline absolute & 54 & 43 & 34 & 24 & 16 & 4 & 1 &. & 1 & 177 \\
relative & 10.8 & 8.6 & 6.8 & 4.8 & 3.2 & 0.8 & 0.2 &. & 0.2 & 35.4 \\
\hline
\end{tabular}


For the $\tilde{0} 0$ YP, a regression $\log N$, m, was deduced in agreement with the polynomial expression

$$
\log X=\sqrt{a(8.1 \check{5}-m)-b}
$$

accepting Gutenberg and Richter's suggestion for the limit $M=9$ and the formula $m=0.62 M+2.5$, which gives as maximum $m=$ 8.15. In EF was commented such a regression, and also that a linear adjustment is only acceptable as a rough approximation and without validity for great values of magnitude. For the $50 \mathrm{YP}$, the old material was used evaluating through the CSE's formula (*), which relates I and $M$ or $m$. But here, for 1961-65, we have five years of magnitude computed from body waves and the regression $\log N, m$, neither is linear. After several trials made, using

$$
\left.\begin{array}{l}
y=A x^{2}+B x+C \\
y=\log N \\
x=\sqrt{(8.15-m)}
\end{array}\right\}
$$

the parameters were deduced by means the least square method, and

$$
\left.\begin{array}{ll}
A=-1.42103 \pm 0.261551 & (12 \%) \\
B=+8.1890 \pm \pm 0.891998 & (10.9 \%) \\
C=-9.67845 \pm 0.003195 & (0.3 \%)
\end{array}\right\}
$$

for $4.0 ₹ m \gtrless 6.0$, refusing frequency corresponding to $m=5.5$ because it is evident its great deviation. The curve has been drawn extrapolating towards $m=4.5$ and $m=6.5$ in a dash line. The solution found satisfied the dita, and thus it is sufficiently clear that regression $\log N, m$, is no linear but polynomial for our area, as Fig 5 shows.

Nevertheless, it is useful a linear simple approximated regression in order to reach rough evaluations. Taking into account that $m=4.19$ is the average of magnitude during 1961-65, and also the grood agreement, for $4.25 \gtrless m \gtrless 4.75$, introducing the parameters in [3] we have

$$
\log N=4.023-0.723 m
$$

available formula to apply it in order to use "expectancy and ex-

(*) European Seismological Commission. 
pectant" $\left.{ }^{*}\right)$. Since expectancy was defined as the value of $m$ for $X=10$, using [5], we have

$$
\begin{array}{lll}
\text { absolute expectancy } & e_{a}=4.1812 & \text { for the whole area } \\
\text { mean expectancy } & e_{n}=3.0512 & \text { for each } 10^{4} \mathrm{sq} \mathrm{km}
\end{array}
$$

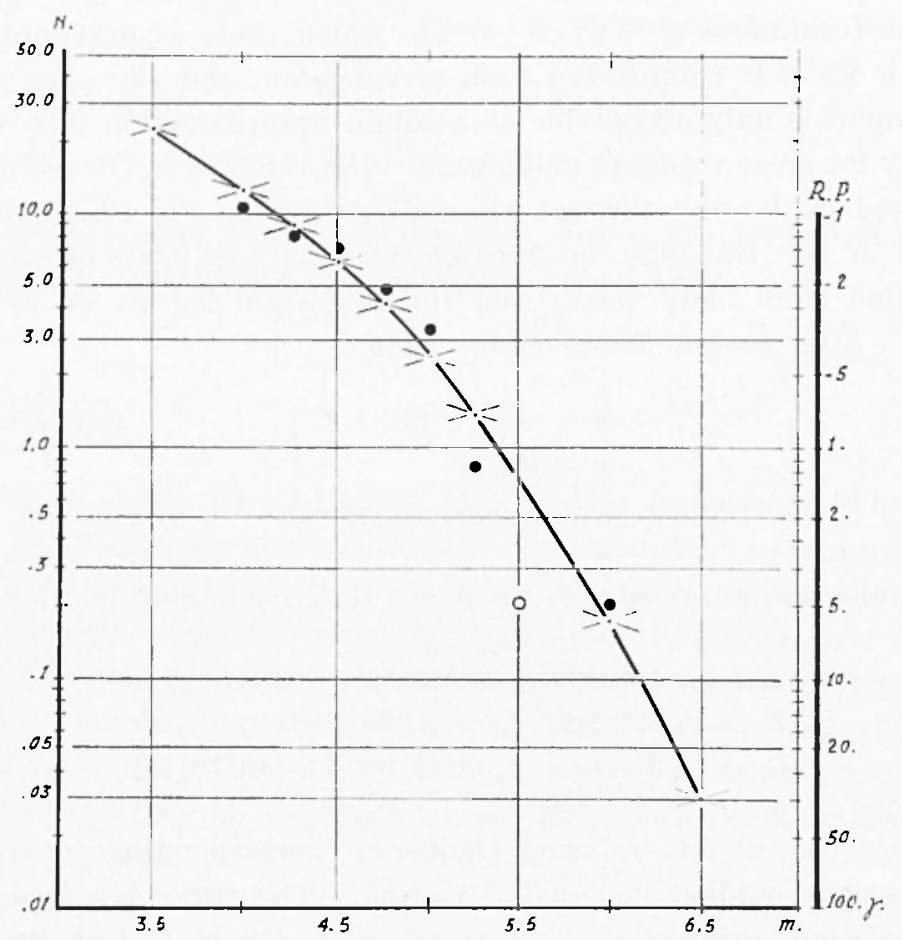

Fig. 5 - Regression $\log _{10} N$ versus $m, 1961-65$.

Comparing these results with those evaluated for the 50 YP $\left(e_{a}=4.852\right)$ it is reasonable to assume that they were higher regarding magnitude, as was discussed in ES, specially lespect the most important shocks No. 2881, 2898 and 3052. In other hand, the first adjustment was made establishing steps of $1 / 2 \mathrm{~m}$, and here $\mathrm{m}$ has been groupped in $1 / 4 \mathrm{~m}$. Consequently, the old information should be displaced about a half unity, less or more, towards the lower $m$ values. Obviously, until we have sufficient material with a well known error for the epicen-

(*) See $\mathrm{EF}$. 
ter determination it is not possible to draw the graphs for the convenient two different regressions: both shallow and intermediate shorks.

Table VI shows the mean magnitude for located shocks since 1916 up to 1965, after having corected the corresponding values for the prior 45 years, substracting $1 / 2 \mathrm{~m}$. So, you can see that mean magnitude is almost constant.

Table VI - Mean values dURINg the last 50 years.

\begin{tabular}{|c|c|c|c|c|}
\hline Period & Annual energy & $\begin{array}{c}\text { Mean } \\
\text { masnitude } \\
\text { fromi steps .5m }\end{array}$ & $\begin{array}{c}\text { Maximal } \\
\text { magnitude } \\
\text { from } \\
\text { form 171 }\end{array}$ & $\begin{array}{c}\text { Locaterl } \\
\text { shocks }\end{array}$ \\
\cline { 2 - 4 } $1916-20$ & $3.20 \times 10^{19}$ & 4.18 & 5.4 & 29 \\
$1921-25$ & $2.60 \times 10^{20}$ & 4.11 & 5.7 & 31 \\
$1926-30$ & $2.70 \times 10^{20}$ & 4.28 & 5.7 & 39 \\
$1931-35$ & $2.03 \times 10^{19}$ & 4.02 & 5.3 & 24 \\
$1936-40$ & $1.32 \times 10^{19}$ & 4.07 & 5.2 & 20 \\
$1941-45$ & $2.40 \times 10^{19}$ & 4.11 & 5.7 & 42 \\
$1946-50$ & $4.15 \times 10^{10}$ & 4.08 & 5.4 & 44 \\
$1951-55$ & $2.78 \times 10^{23}$ & 4.22 & 6.8 & 63 \\
$1956-60$ & $5.06 \times 10^{21}$ & 4.35 & 6.1 & 64 \\
$1961-65$ & $4.41 \times 10^{20}$ & 4.19 & 5.8 & 177 \\
\hline
\end{tabular}

7. Sisismic Fidux.

Following the same criterion and the method which was explained in ES, seismic or tectonic flux has been computed by means the formula

$$
\varnothing=\log F^{H}=0.5 \log E_{T}-1.097
$$

where $E_{T}$ is the total encrgy released during 1961-65 into each seismiaally active quadricle (see Table III). Fig. 6 shows these results in order to be able to check them to those presented in ES.

The maximum tectonic flux is just in B05 $(\varnothing=9.6)$ Zone II, Region 18, in Southern area. Energy has been computed using

$$
\log E=4.1+28.6 m
$$




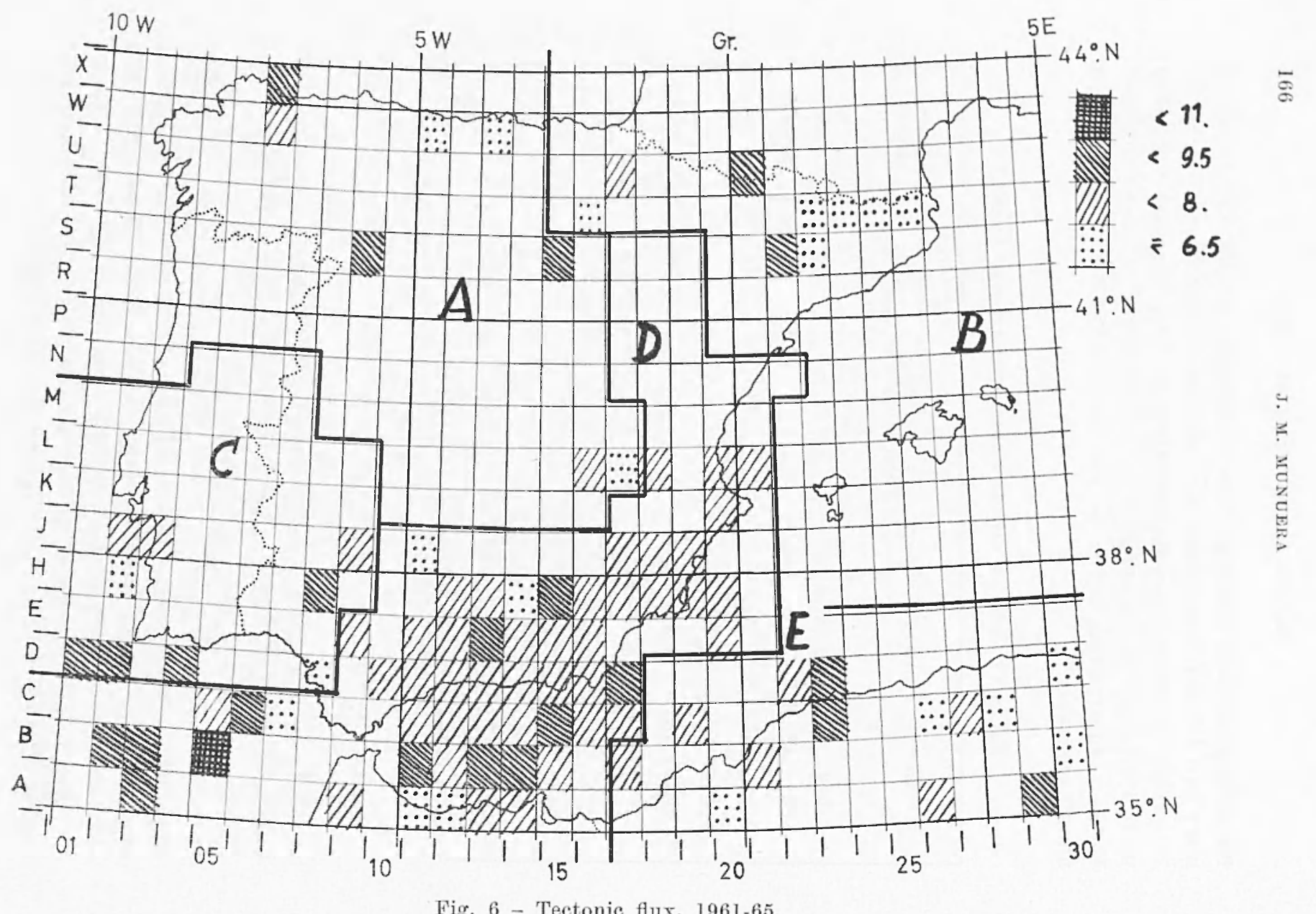


Assuming mean magnitude, the error for the energy release eraIuation up to $1960 \mathrm{is,} \mathrm{more} \mathrm{or} \mathrm{less,} \mathrm{the} \mathrm{difference} \mathrm{between} \log E=3.79$ $+2.65 m$ and $\log E=4.14+2.86(m-0.5)$; that is, about 0.11 to add to $\log E$; a value lower than the probable error in magnitude. Thus, it is unnecesary to modify the total amount estimated in ES as the energy released during 160 years, which was $1.5 \times 10^{-1}$ erg, approximately.

Table VI lists also the estimated seismic energy released for the located shocks 1916-65; the annual seismic energy released in 1961-65 is detailed in Table III, for the different zones and regions on the investigated area. Total seismic energy released during the last five years is $2.2 \times 10^{2 \mathrm{l}} \mathrm{erg}$, approximately, or $4.41 \times 10^{20}$ erg per year, which represents the $4.5 \%$ of the average during the preceding 160 year lapse. In conclussion, the present period, 1961-65, is accumulative, as it was expected from the shape of the Benioff's curve which was drawn in ES.

\section{Comments.}

The above results, speaking in a general sense, agree with the preliminaly ones for the $50 \mathrm{YP}$. Seismic activity is unquiet on the area, but $n 0$ mystifying, though its features of frequency and tectonie flux move from year to year. The migration of maximal density is happening since more than one and a half century ago and probably even before. Table VII summarized the main character dedueed from the tectonic flux distribution; the intralpine character is emphasized by the ratio 1/II (1.96 for the 50 YP and 3.0 for 1961-65). Active quadricles are, respectively, $38,1 \%$ for the 50 YP and $18.4 \%$ for 1961-65; so we caln say that the present period is accumulative. Migration is also confremed because the particular seismicity of the 50 YP inside the Pyrenees Oval was $17.2 \%$ rersus total seismicity inside the Alpine Geosyncline, but it is just $4.2 \%$ for the lapse 1961-65. Intermediate shocks (preliminary determinations) in 1961-65 agree with the great active laults, in the same way than those shocks up to 1960, (see ES).

\section{ACKNOWLEDGEMENT.}

This research has been sponsored by the $\Delta$ ir Force Office of Scientific Research, $\mathrm{OAR}$, through the European Office of Aerospace Rescarch, 
Table VII - CharaCter of SEIsMicity From the teCtonic Flux DISTRIBUTION ON THY AREA.

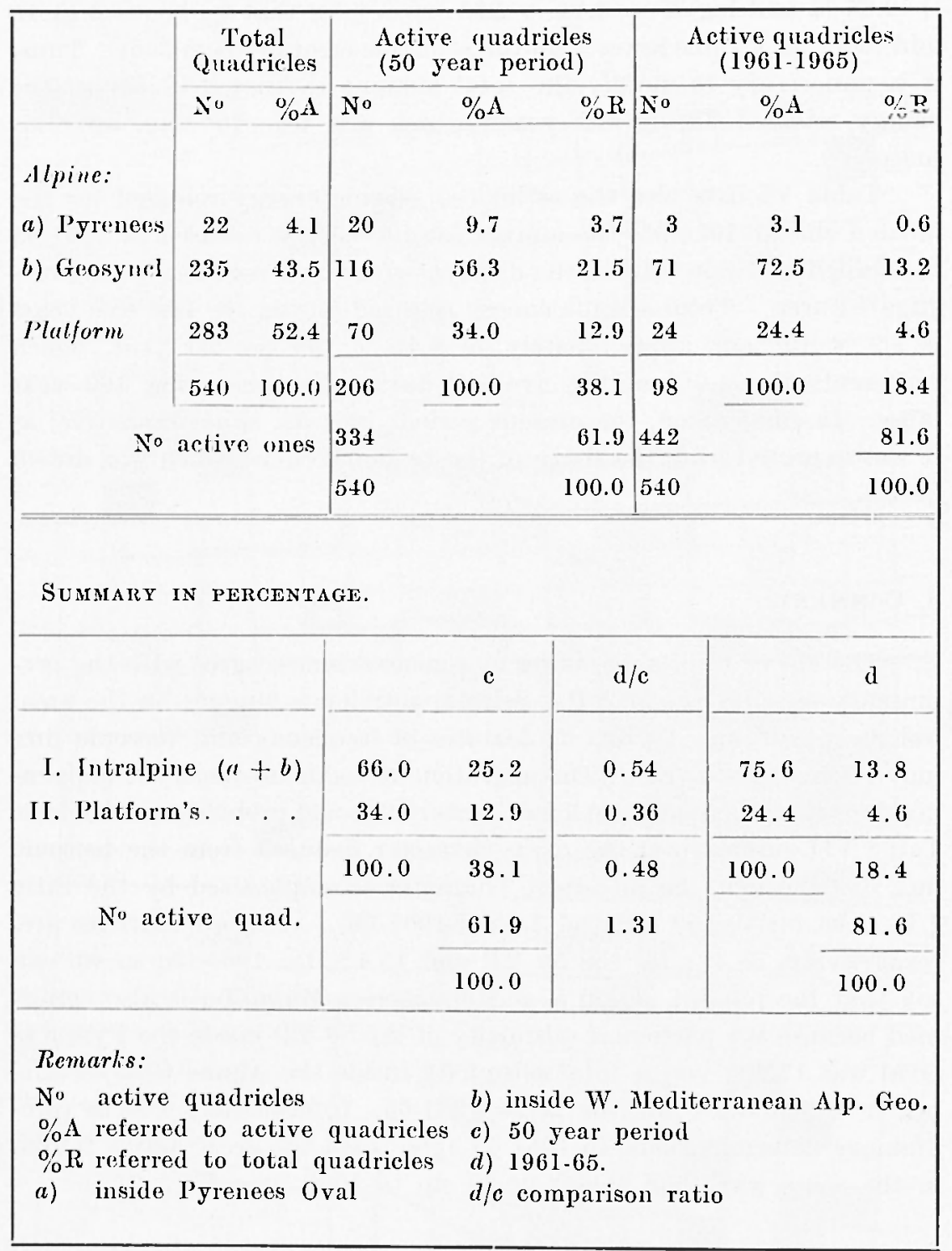

EOAR, according the Contract $A F$ $61(052)-657$, as part of the Advanced Research Project Agency's Project VEIA-UNIFORM, of the U.S. Air Force. 


\section{REFERENCES}

Munuera J. M., Caracteres mecánicos de los sismos, URANIA, 255-256, Tarragona. (CMI).

- Seismic Data, "Memorias del Instituto Geográfico y Catastral " (MIGC), XXXII, 1, (Madrid, 1963). (SD).

- Epicenters and Frequency, “MIGC", XXXIII, 4, (Madrid, 1964). (EF).

- A Seismic Probability Map, "Annali di Geofisica" (AG) XVII, 4, (Roma, 1964). (PMI).

- Energy release and Seismic Flux, "AG", XVIII, 3, (Roma, 1965). (ES).

- Seismic MLagnitude and Specific Absorption Coefficient in the Iberian Region, (to be published). (MA).

Munuera J. M. and Udias S.J., Programa de cálculo electrónico para el ajuste de las delerminaciones epicentrales de sismos próximos, presented to Coloquio de Sismología, Madrid, April, 1966, (to be published). (ECP). 